\title{
Regulación emocional de los resultados adversos en competición. Estrategias funcionales en deportes colectivos
}

\author{
Emotional regulation in team sports: An approach to the \\ modulation of emotional states in competitive situations
}

\section{Regulação emocional em esportes coletivos: uma abordagem para a modulação de estados emocionais em situaçóes competitivas}

\author{
Xavier Oriol Granado ${ }^{1}$, Miquel Gomila Andreu ${ }^{2}$ y Gemma Filella Guiu ${ }^{3}$ \\ 1 Universidad Autónoma de Chile, 2 INEFC Lleida, 3 Universidad de Lleida
}

\begin{abstract}
Resumen: Este estudio pretende analizar cuáles son las emociones más experimentadas por los jugadores ante resultados desfavorables en un partido, si existe un uso diferencial de las estrategias de regulación usadas dependiendo de la emoción experimentada y analizar la funcionalidad de las diferentes estrategias que tanto los jugadores a nivel individual y grupal como el entrenador utilizan para regular estas experiencias emocionales. Para el estudio se seleccionaron 8 equipos ( 4 femeninos y 4 masculinos), todos ellos de categorías profesionales, de diferentes disciplinas deportivas: Futbol, baloncesto, hockey patines y balonmano. A todos los jugadores, se les administró una entrevista semiestructurada para conocer el tipo de emociones experimentadas ante situaciones desfavorables, las estrategias de regulación emocional utilizadas y la valoración de la funcionalidad de las mismas. También se administró el cuestionario de regulación emocional (ERQ). Para el análisis de los datos cualitativos se realizó un proceso de categorización inductiva con posteriores análisis inferenciales y para el análisis cuantitativo del cuestionario se realizaron pruebas $(\mathrm{T})$ para una misma muestra. Los resultados muestran la conexión existente entre el tipo de emoción experimentada ante los resultados adversos y el uso diferencial de unas u otras estrategias de regulación. Se observó que la reevaluación cognitiva y el contagio emocional positivo grupal son las estrategias percibidas como más funcionales ante estas situaciones.

Palabras clave: Regulación emocional, estrategias funcionales, rendimiento deportivo, resultados adversos

Abstract: This study analyzes what are the most common emotions of players in unfavourable results in a match and if there are a differential use of regulation strategies used depending on the emotion experienced. We analyze the functionality of the different strategies that players both individually and collectively and the coach use to regulate these emotional experiences. For the study we selected 8 teams ( 4 female and 4 male) professional categories corresponding to different sports: Soccer, basketball, hockey and handball. All players responded a semi-structured interview about the type of emotions
\end{abstract}

experienced in unfavourable situations, emotional regulation strategies used and the valuation of the feature's functionality. Also we administered a questionnaire of emotion regulation strategies (ERQ). The qualitative data analysis was performed with inductive categorization process and the subsequent inferential analysis. For the quantitative analysis we used testing $(T)$ for the same sample. The results showed the connexion between the type of experimented emotion in unfavourable results and the differential use of regulation strategies. It was found that cognitive reappraisal and positive sharing of emotions are the most functional perceived strategies in this situations.

Key words: Emotional regulation, functional strategies, performance in sport, unfavourable results

Resumo: Este estudo analisa quais são as emoçốes mais comuns de jogadores em resultados desfavoráveis em uma partida e se há um uso diferencial de estratégias de regulação utilizadas Dependendo da emoção experimentada. Analisamos a funcionalidade das diferentes estratégias que os jogadores tanto individual e coletivamente eo uso treinador para regular essas experiências emocionais. Para o estudo, foram selecionados oito equipes (4 mulheres e 4 homens) categorias profissionais correspondentes a diferentes esportes: futebol, basquete, hóquei e andebol. Todos os jogadores responderam uma entrevista semi-estruturada sobre o tipo de emoçôes com experiência em situaçôes desfavoráveis, as estratégias de regulação emocional utilizadas ea valorização da funcionalidade da função. Também aplicado um questionário de estratégias de regulaçấo da emoção (ERQ). A análise qualitativa dos dados foi realizada com o processo de categorização indutiva e análise inferencial subseqüente. Para a análise quantitativa, utilizou-se o teste $(\mathrm{T})$ para a mesma amostra. Os resultados mostraram a relação entre o tipo de emoção experimentada em resultados desfavoráveis e do uso diferencial de estratégias de regulação. Verificou-se que a reavaliação cognitiva e partilha de emoçóes positivas são as estratégias mais funcionais percebidos nesta situaçóes.

Palavras chave: Regulação emocional, estratégias funcionais, o desempenho no esporte, resultados desfavoráveis

\section{Introducción}

La importancia de las emociones en el rendimiento deportivo se fundamenta principalmente en los estudios del afron-

Dirección para correspondencia [Correspodence address]: Xavier Oriol Granado. xoriol@pip.udl.cat tamiento del estrés (Hardy, Mullen y Jones, 1996; Lazarus, 1999) y el modelo de zona óptima de desarrollo IZOF basado en la identificación de los estados emocionales en el rendimiento individual (Hanin, 1980, 1986, 1997, 2000b, 2000c). 
Los estudios sobre el estrés en el deporte se han centrado en el estrés sufrido por los deportistas en las situaciones precompetitivas (Kroll, 1980; Gould y Weinberg, 1985; Feltz, Lirgg y Albrecht, 1992) durante la situación competitiva (Jones y Hardy, 1990) o después de la competición (Pargman, 1986).

Las situaciones estresantes durante el desarrollo de las competiciones deportivas puede producir alteraciones psicológicas tales como la capacidad de concentración, una pérdida del foco de atención o un incremento de la ansiedad, la frecuencia cardíaca y la tensión muscular (Márquez, 2004; Estrada y Pérez, 2008). Lazarus (1999) enfatizó la importancia que la evaluación de la situación tenia sobre la calidad y la intensidad de las emociones y el efecto que a su vez este proceso tenia sobre el rendimiento deportivo. Las diferentes teorías sobre la evaluación de las emociones sugeridas en primer término por Arnold (1960) y Lazarus (1968) explican que las emociones surgen de la evaluación subjetiva de un sujeto ante un estímulo o situación determinada (Weiner, 1982, 1986; Roseman, 1984; Smith y Ellsworth, 1985; Frijda, 1986; Lazarus, 1991; Scherer, 1997). El tipo de evaluación por lo tanto de la situación determina la emoción que se experimenta y en consecuencia la intensidad de la experiencia emocional (Gross y Thompson, 2007; Siemer, Mauss y Gross, 2007).

Las emociones tienen una función adaptativa en situaciones de estrés (Folkman y Lazarus, 1985; Frijda, 1986; Levenson, 1988) y algunos estudios han focalizado su atención en como las emociones positivas y negativas influyen en el afrontamiento del estrés en una situación determinada (Zautra, 2003; Zautra, Affleck, Tennen, Reich y Davis, 2005). Las emociones negativas como la ira o la ansiedad influyen negativamente sobre las relaciones sociales (Lazarus, 1996) y la aparición de trastornos cardiovasculares (Palmero, Díez y Breva, 2001; Siegman y Smith, 1994). A estos se le añaden los trastornos de tipo afectivo y de tipo depresivo (Beck, 1976) entre otros.

Las emociones positivas pueden contribuir a la resistencia y a la recuperación al estrés y pueden interrumpir la experiencia de emociones negativas mientras se produce una situación estresante (Fredrickson y Joiner, 2002). Inducir emociones positivas implica cambios en los patrones de pensamiento e implica una mayor eficiencia en las tareas (Isen y Means, 1983; Isen Rosenzweig y Young, 1991). La necesidad de inducir emociones positivas y disminuir las emociones negativas conlleva el desarrollo de estrategias de regulación emocional con el objetivo de mejorar el rendimiento deportivo.

De acuerdo con numerosos autores podemos entender la regulación emocional como la modulación (aumentar, mantener o disminuir) de cualquier aspecto de una respuesta emocional, incluyendo experiencia y comportamiento expresivo (Gross, 1998; Gross y John, 2003; Eisenberg y Spinrad, 2004; Goldsmith y Davidson, 2004).
Existen numerosos modelos de regulación emocional (Bonanno, 2001; Forgas y Ciarocchi, 2002; Gross y Thompson, 2007, entre otros). De entre ellos destacamos el modelo de Gross y Thompson (2007) que pone énfasis en dos estrategias principales de regulación emocional. Por un lado, la reevaluación cognitiva que se basa en un cambio a nivel cognitivo que modifica el impacto emocional y la supresión cognitiva que implicaría una inhibición de la respuesta emocional. Los mismos autores han estudiado las consecuencias de una y otra estrategia concluyendo que la reevaluación cognitiva tiene importantes beneficios en la disminución de síntomas de depresión, mayor autoestima y satisfacción con la vida y mejores relaciones sociales, los que utilizan la supresión como estrategia enmascaran sus sentimientos obteniendo un estado de ánimo más negativo, la experimentación de más emociones negativas y un mayor nivel de estrés (Gross, 1998; Richards y Gross, 2000; Gross y John, 2003).

La regulación emocional debe entenderse desde una concepción intra e interpersonal (Rimé, 2009). Y ello resulta especialmente necesario en los deportes de equipo. La relación que se establece por lo tanto con las otras personas tiene importantes repercusiones en el proceso de regulación. Rimé (2009) enfatiza la importancia del contagio emocional en los procesos de regulación interpersonal. El contagio emocional implica beneficios en el afecto positivo y además permite establecer mayores vínculos sociales.

En primer lugar, este estudio pretende evaluar cuales son las estrategias de autorregulación y heteroregulación más usadas por los jugadores ante resultados adversos y si existe un uso diferencial de estas estrategias en función de la emoción experimentada. También observar cuales son las estrategias más utilizadas por el grupo y por el entrenador ante este tipo de situaciones.

En segundo lugar, observar la funcionalidad percibida por parte de los jugadores de las estrategias de autorregulación y heteroregulación, las estrategias de regulación que usa el equipo y las estrategias que utiliza el entrenador para regular las experiencias emocionales ante resultados adversos.

\section{Método}

\section{Participantes}

Para el estudio se seleccionaron 7 equipos ( 4 femeninos y 4 masculinos) de categorías profesionales correspondientes a diferentes disciplinas deportivas; Futbol, baloncesto, hockey patines y balonmano. En total la muestra está compuesta por 112 sujetos, con una distribución por sexos de 61 jugadores masculinos y 51 jugadoras femeninas (tabla 1 ). 
Tabla 1. Datos descriptivos. Modalidades deportivas y media y desviación típica de la edad.

\begin{tabular}{llll}
\hline Modalidad Deportiva & & Categoría & Edad \\
\hline Baloncesto & Masculino & EBA & $23.2(1.33)$ \\
& Femenino & Liga EBA Femenina & $21.4(1.09)$ \\
Hockey patines & Masculino & Primera Nacional Española & $45.6(2.02)$ \\
& Femenino & Primera Catalana & $19(0.74)$ \\
Balonmano & Masculino & División de Honor de Plata & $24(1.27)$ \\
& Femenino & Primera catalana & $19(0.74)$ \\
Fútbol & Masculino & Segunda División B & $25(2.12)$ \\
& Femenino & Segunda División Femenina & $23(1.86)$ \\
\hline
\end{tabular}

\section{Instrumentos}

Para la recogida de datos se utilizaron dos instrumentos. El primero de ellos consistía en una entrevista semiestructurada donde se pedía a los jugadores mediante una pregunta abierta que especificaran el tipo de emoción experimentada ante un resultado en contra y las estrategias de regulación utilizadas tanto a nivel individual, a nivel grupal (equipo), así como las estrategias utilizadas por el entrenador. En la misma entrevista también se pedía a los jugadores la valoración de cada estrategia mediante una escala likert (escala oscilando entre $1=$ nada satisfactoria y $10=$ En totalmente satisfactoria).

Se administró también el cuestionario de Regulación Emocional ERQ (Gross y John, 2003) adaptado al castellano por Cabello, Fernández-Berrocal, Ruiz-Aranda y Extremera (2006).

Este cuestionario permite evaluar las diferencias que se producen entre las estrategias de reevaluación cognitiva (en qué medida las personas suelen intentar cambiar a nivel cognitivo 1 el impacto emocional que determinada situación les genera) y la estrategia de supresión (en que medida la persona opta por inhibir su comportamiento expresivo) en base a 10 ítems (escala oscilando entre $1=$ En desacuerdo total y $7=$ En total acuerdo).

\section{Procedimiento}

Para llevar a cabo el estudio se contactó con el staff técnico de los diferentes equipos deportivos para llevar a cabo la recogida de datos correspondiente. Se elaboró un protocolo para la pasación de los cuestionarios que fueron aplicados siempre por el mismo investigador. Antes del entrenamiento, se explicaba a los jugadores el objetivo del estudio y a continuación se daban las instrucciones oportunas para cumplimentar los instrumentos. Primero se administró la entrevista semiestructurada y a continuación el cuestionario de regulación emocional EQR (Gross y John, 2003) que fueron respondidos individualmente y de forma anónima.

\section{Análisis de datos}

El análisis cualitativo procedente de las entrevistas se realizó mediante un proceso de categorización inductiva usando matrices de análisis descriptivas. Así pues, se procedió a establecer unas categorías referentes a las diferentes estrategias usadas por el jugador: (autorregulación: Estrategias que el jugador usa para regular su propia emoción y heteroregulación: Estrategias que el jugador usa para regular las emociones de los compañeros), estrategias de regulación usadas por el equipo y estrategias de regulación usadas por el entrenador. Cabe destacar que para crear las diferentes categorías correspondientes al tipo de estrategias se tuvo en cuenta también los modelos de regulación emocional existentes (tabla 2).

Tabla 2. Estrategias de regulación individual, grupal y del entrenador

\begin{tabular}{l}
\hline Estrategias de regulación individuales \\
\hline Estrategias usadas para regular su propia emoción (Autorregulación) \\
Reevaluación cognitiva \\
Supresión \\
Estrategias para disminuir la reactividad \\
Buscar una solución \\
No regula (Se enfada, contagio emocional neg. a los demás) \\
Estrategias donde el jugador buscar regular las emociones de los demás (Heteroregulación) \\
Contagio emocional (hacia el resto del equipo) \\
Buscar solución conjunta (con el resto de los compañeros) \\
\hline
\end{tabular}




\begin{tabular}{l}
\hline Estrategias de regulación grupales \\
\hline Contagio emocional positivo \\
Supresión grupal \\
No regulan (Individualidad, contagio emocional negativo) \\
Estrategias para disminuir la reactividad grupal \\
Buscar solución conjunta \\
\hline Estrategias de regulación usadas por el Entrenador \\
\hline Contagio emocional positivo \\
Estrategias para disminuir la reactividad \\
No regula (grita, contagio emocional negativo). \\
Cambia la situación de juego
\end{tabular}

El análisis de las matrices se realizó mediante el paquete estadístico SPSS 17. Se realizaron análisis descriptivos y análisis inferenciales. Se procedió a evaluar el cumplimiento de los supuestos para la utilización de pruebas paramétricas, a saber, normalidad, homeostaicidad e independecia (Balluerka y Vergara, 2002). Los resultados mostraron que se cumplían los supuestos. Se realizaron comparaciones de medias mediante análisis de la varianza de un factor con comparaciones múltiples de tipo Scheffé para observar la relación entre la satisfacción de las estrategias de regulación empleadas. También se realizaron tablas de contingencia para observar la relación entre el tipo de emoción y las estrategias. Para el análisis cuantitativo de la información procedente del cuestionario se realizó una comparación de medias (T) para una misma muestra.

\section{Resultados}

Uso de estrategias de regulación ante resultados adversos

En primer lugar, se analizaron las diferencias existentes ante resultados adversos entre el uso de la estrategia de reevluación y la de supresión que contempla el cuestionario ERQ. Los resultados referentes a la consistencia interna del cuestionario en el total de la muestra de sujetos mostraron un coeficiente alfa de Cronbach de 0.79 para la estrategia de reevaluación cognitiva y de 0.86 para la estrategia de supresión.

En la tabla 3 se observan los resultados referentes a las diferencias existentes entre las dos estrategias que evalúa el ERQ: reevaluación cognitiva $(M=4.34)$ y supresión $(M=3.67)$. Se observaron diferencias significativas a favor de la reevaluación $(t=3.67, g l=111, p>.001)$.

Tabla 3. Medias y desviación típica de las estrategias de reevaluación y supresión ante resultados adversos

\begin{tabular}{llll}
\hline Estrategias de regulación & $\mathrm{N}$ & Media & $\mathrm{DT}$ \\
\hline Reevaluación cognitiva & 112 & 4.34 & 1.09 \\
Supresión & 112 & 3.67 & 1.08 \\
\hline
\end{tabular}

Los resultados referentes al uso de estrategias ante este tipo de situación (mediante la categorización inductiva), muestran que la estrategia más usada por parte de los jugadores (tabla 2) ante un resultado adverso es la estrategia de reevaluación cognitiva (autorregulación) $(M=50.5)$, seguida del contagio emocional positivo hacia los compañeros (heteroregulación) $(\mathrm{M}=32.1)$ (tabla 4)

Resultados referentes al tipo de emoción experimentada y el uso diferencial de estrategias de autor y heteroregulación por parte del jugador

Los resultados referentes al tipo de emoción experimentada muestran que las emociones que más experimentan los jugadores ante un resultado desfavorable, son la emoción de esperanza (41.3\%) seguida de la emoción de ansiedad (17.4\%), la de ira (13.8\%) y aversión (11\%). En el análisis Chi cuadrado realizado respecto al resultado derivado de la comparación entre el tipo de emoción y las estrategias de regulación emocional utilizadas, la esperanza muestra diferencias significativas a favor de la reevaluación cognitiva $(z=2.8 ; p<.05)$ y la aversión presenta diferencias a favor de la estrategia de buscar una solución $(z=2.5 ; p<.05)$.

Resultados referentes al uso de estrategias de regulación emocional grupales y a las utilizadas por el entrenador ante resultados adversos

En lo que respecta a las estrategias de regulación más utilizadas a nivel grupal ante un resultado desfavorable, destacan el contagio emocional positivo $(\mathrm{M}=63.3)$ y la no regulación $(M=22.9)$. Esta última implicaría no afrontar la situación grupalmente y por lo tanto se produciría un afrontamiento individual de la situación. En las estrategias de regulación emocional por parte del entrenador, destacan especialmente tres estrategias. La primera corresponde al contagio emocional hacia los jugadores $(M=52.3)$, la no regulación $(M=21.1)$ y finalmente la estrategia referente a cambiar la situación de juego $(M=18.3)$ (tabla 4). 
Funcionalidad percibida de los jugadores en el uso de estrategias de auto y heteroregulación, de regulación grupal y las usadas por el entrenador ante resultados adversos

Las estrategias de regulación emocional usadas que son consideradas como más funcionales por los jugadores (Tabla 5) son las de reevaluación cognitiva (autorregulación) ( $M=7.49)$, contagio emocional positivo hacia los otros (heteroregulación) $(\mathrm{M}=7.37)$ y la de buscar una solución conjunta con los demás compañeros (heteroregulacion) $(\mathrm{M}=7.25)$. Por el contrario, la estrategia de supresión (autorregulación) es la única que obtiene una valoración inferior al $5(\mathrm{M}=4.33)$.

En el análisis de varianza se observaron diferencias en la valoración de las estrategias de regulación individuales $(F=$ $10.248 ; g l=5,89 ; p=.000)$. En el análisis de comparaciones múltiples entre las medias de las diferentes estrategias, se observan diferencias significativas. La reevaluación cognitiva obtiene puntuaciones superiores en comparación con la supresión $(p<.001)$ y las estrategias de reactividad ( $p=.018)$. Las estrategias de supresión obtienen puntuaciones inferiores respecto a las estrategias de reactividad $(p=.016)$ y a las estrategias referentes a buscar una solución $(p=.001)$ y contagio emocional hacia los demás $(p<.001)$.

En cuanto a la funcionalidad percibida de las estrategias grupales, las más valoradas son las de contagio emocional positivo $(\mathrm{M}=8.07)$, las referentes a buscar una solución conjunta $(\mathrm{M}=7.5)$ y las estrategias de disminución de la reactividad $(\mathrm{M}=7)$. El análisis de varianza mostró diferencias entre la valoración de las estrategias grupales $(F=33.311 ; g l=4$, $89 ; p=.000)$. Las comparaciones múltiples mostraron mayores puntuaciones en la estrategias de contagio emocional respecto a la no regulación $(p<.001)$. También se observaron puntuaciones superiores en las estrategias de regulación de la respecto a la no regulación $(p=.010)$ y se observaron diferencia a favor de buscar una solución conjunta respecto a la no regulación $(p<.001)$.

Los resultados del análisis referente a las estrategias de regulación utilizadas por el entrenador muestran que las que obtienen una mayor valoración de la satisfacción son el cambio de la situación de juego $(M=8.3)$, seguida por el contagio emocional positivo $(\mathrm{M}=7.8)$ y la regulación de la reactividad (7.1). El análisis de varianza también mostró diferencias respecto a la valoración en las estrategias del entrenador $(F=$ 11.448; $g l=3$, 99; $p=.000)$. El análisis de comparaciones múltiples entre medias mostró diferencias significativas a favor del contagio emocional positivo respecto a la no regulación $(p<.001)$ y puntuaciones también significativamente superiores en el cambio de la situación de juego respecto a la no regulación $(p<.001)$.

Tabla 4. Medias y desviación típica de las estrategias de regulación emocional individuales, grupales y colectivas.

\begin{tabular}{llll}
\hline Estrategias usadas por el jugador & $\mathrm{N}$ & Media & $\mathrm{DT}$ \\
\hline Reevaluación cognitiva & 55 & 7.49 & 1.03 \\
Supresión & 6 & 4.33 & 4.33 \\
Estr. de disminuir la reactividad & 9 & 6.22 & 1.48 \\
Buscar una solución conjunta & 8 & 7.25 & .95 \\
Contagio emocional positivo (hacia el resto) & 35 & 7.37 & .94 \\
\hline Estrategias Grupales & $\mathrm{N}$ & Media & $\mathrm{DT}$ \\
\hline Contagio emocional positivo & 69 & 8.07 & 1.27 \\
No regulación & 25 & 3.80 & 2.55 \\
Estr. de disminución reactividad & 3 & 7.00 & 1.00 \\
Buscar una solución conjunta & 10 & 7.50 & .97 \\
\hline Estrategias del entrenador & $\mathrm{N}$ & $\mathrm{Media}$ & $\mathrm{DT}$ \\
\hline Contagio emocional positivo & 57 & 7.82 & 1.32 \\
Estr. de disminución reactividad & 9 & 7.11 & 1.05 \\
No regulación & 23 & 6.13 & 1.79 \\
Cambiar la situación & 20 & 8.30 & 7.50 \\
\hline
\end{tabular}


Tabla 5. Diferencias significativas en la funcionalidad percibida de las estrategias individuales, grupales y del entrenador (análisis de comparaciones múltiples de tipo Scheffé)

\begin{tabular}{lllll}
\hline Funcionalidad percibida de las estrategias de autorregulación & Diferencia de medias & Error típico & Sig. \\
\hline Reevaluación Congitiva & Supresión emocional & $3.158\left(^{*}\right)$ & .484 & .000 \\
& Estr. de reactividad & $1.269\left(^{*}\right)$ & .404 & .018 \\
Supresión & Estr. de reactividad & $-1.889\left(^{*}\right)$ & .593 & .016 \\
& Buscar una solución & $-2917\left(^{*}\right)$ & .726 & .001 \\
& Contagio emocional & $-3-038\left(^{*}\right)$ & .497 & .000 \\
\hline Funcionalidad percibida de las estrategia (grupales) & Diferencia de medias & Error típico & Sig. \\
\hline Contagio emocional & No regulación & $4.265\left(^{*}\right)$ & .382 & .000 \\
No regulación & Estr. de reactividad & $-3.200\left(^{*}\right)$ & 1.001 & .010 \\
Buscar una solución (conjunta) & No regulación & $3.700\left(^{*}\right)$ & .613 & .000 \\
\hline Funcionalidad percibida de las estrategias usadas por el Entrenador & Diferencia de medias & Error típico & Sig. \\
\hline Contagio emocional & No regulación & $1.694\left(^{*}\right)$ & .337 & .000 \\
No regulación & Cambio de situación de juego & $-3.700\left(^{*}\right)$ & .613 & .000 \\
\hline
\end{tabular}

* La diferencia entre medias es significativa al nivel 0.05

\section{Discusión}

El presente estudio, pretendía en primer lugar, evaluar cuales son las estrategias de auto y heteroregulación más utilizadas por los jugadores ante una de las situaciones que produce un mayor impacto emocional durante un partido, en concreto, los resultados desfavorables. Y además, observar si existe un uso diferencial en función de la emoción experimentada.

Los resultados muestran en primer lugar que los jugadores usan más la reevaluación cognitiva que la supresión ante resultados adversos. En concreto vemos que la reevaluación cognitiva (autorregulación) es la estrategia más utilizada seguida por el contagio emocional hacia los compañeros (heteroregulación). La reevaluación implica un cambio mental que favorece un menor impacto emocional ante esa situación en concreto (Gross y John, 2003). El uso de esta estrategia de regulación se ha asociado a una disminución de la reactividad fisiológica y se asocia a un mayor intercambio de emociones y un mayor apoyo social (Richards y Gross, 2000).

El contagio emocional positivo hacia los compañeros es una estrategia relacionada con el apoyo social. El hecho de compartir emociones con otras personas y afrontar el hecho de forma constructiva aumenta el bienestar y favorece la percepción de una mayor felicidad (Larsen y Prizmic, 2008).

En lo que respecta a las estrategias de regulación más utilizadas a nivel grupal el contagio emocional positivo es la estrategia más usada seguida de la no regulación. Como ya se ha comentado, el contagio emocional positivo implica compartir la afectividad positiva ante una situación que a priori podría ser considerada como negativa. Encontrar algo positivo en un suceso negativo es una manera de tomar el control sobre la situación o evento (Emmons y Shelton, 2002). El hecho de que los deportistas intenten apoyar a sus compañeros induciendo a un contagio emocional hacia ellos, favorece el clima de confianza entre los miembros del equipo. Además, compartir emociones con otras personas ha demostrado importantes efectos a nivel cognitivo y en consecuencia efectos también a nivel fisiológico (Coan, 2008; Rimé, 2009). Se produce un reparto de la carga emocional (Coan, 2008) y además el compartir emociones positivas implica un proceso de capitalización en el que los episodios emocionales no son vistos como problemas que hay que superar sino como oportunidades (Langston, 1994). La no regulación correspondería a una estrategia de evitación de la situación y ello implica una tendencia a rechazar la realidad de lo ocurrido.

Respecto al uso diferencial de las estrategias en función de la emoción experimentada, observamos que cuando los sujetos valoran la situación adversa con esperanza utilizan más la estrategia de reevaluación cognitiva. Es decir, realizan una nueva construcción mental de la situación adversa y no la valoran como negativa sino que les plantea un reto por ello experimentan esperanza en lugar de otra emoción negativa como podría ser tristeza o ira. Cuando los sujetos experimentan aversión, ello favorece un mayor uso de la estrategia de búsqueda de soluciones. Estos datos indican que el tipo de emoción que experimentan influye de forma muy importante en el uso de un determinado tipo de estrategias de regulación emocional. Este hecho se produce por la importancia que la evaluación cognitiva de las situaciones tiene en la experimentación de una u otra emoción, así como la intensidad en la que se produce dicha emoción (Gross y Thompson, 2007; Siemer, Mauss y Gross, 2007). Los estudios referentes 
a la evaluación de las emociones, han tenido y tienen una importante relevancia debido a la discrepancia existente entre si existen o no patrones generalizables de evaluación. (p. ej. Frijda, Markam, Sato y Wiers, 1995; Scherer, 1997; Mesquita y Ellsworth, 2001).

En el mundo del deporte, tener en cuenta como se evalúan las emociones en las diferentes situaciones que puedan generar emociones negativas en un partido, puede resultar especialmente útil para trabajar la regulación emocional en los entrenamientos. La evaluación que realizan de una emoción en concreto y la intensidad con la que la experimentan es fundamental para reconocer su realidad subjetiva respecto a como estas emociones afectan a su rendimiento (Jones y Hanton, 2001). Esta percepción puede no corresponder con la realidad objetiva y por ello es imprescindible fomentar su confianza a través de una buena gestión emocional. Ante la misma situación, observamos a jugadores que experimentan emociones positivas como sería la esperanza y por el contrario jugadores que experimentan emociones negativas como la ira o la aversión. Ello nos remite a la importancia de la estrecha unión entre cognición y emoción (Damasio, 2010).

El segundo objetivo que nos planteábamos era observar la funcionalidad percibida por parte de los jugadores de las estrategias de autorregulación y heteroregulación, las estrategias de regulación que usa el equipo y las estrategias que utiliza el entrenador para las situaciones producidas ante resultados adversos.

Respecto a las estrategias usadas por el jugador, la reevaluación cognitiva es la estrategia de autorregulación que los jugadores perciben como más funcional ante resultados adversos a diferencia de la supresión que es la considerada como menos funcional. Respecto a las estrategias de heteroregulación, el contagio emocional positivo y la búsqueda de una solución conjunta son las estrategias consideradas como más funcionales. La mayoría de estudios centrados en la regulación emocional lo han hecho des de un punto de vista intrapersonal. Sin embargo, la mayoría de las experiencias emocionales se producen con otras personas y además es indudable que intentamos ejercer una influencia sobre la experiencia emocional de los demás y de igual manera los demás influyen sobre nuestras experiencias emocionales (Rimé, 2012). Los deportes colectivos son además experiencias grupales que hacen todavía más necesario tener en cuenta la regulación desde un punto de vista intra e interpersonal.

Respecto a las estrategias grupales, coinciden el contagio emocional positivo y la búsqueda de una solución conjunta como estrategias funcionales ante los resultados adversos. La estrategia de buscar una solución conjunta, sería también de algún modo una forma de compartir las emociones, pero la principal diferencia entre estas dos estrategias recae en la implicación afectiva. Rimé (2009) destaca la importancia que el nivel cognitivo, pero también el afectivo tienen en la regula- ción interpersonal de las emociones. Resulta muy importante establecer objetivos comunes entre el equipo, pero en demasiadas ocasiones, se pasa por alto la importancia que la implicación afectiva y el clima emocional de confianza entre ellos tiene en la regulación de los episodios emocionales. Finalmente la disminución de la reactividad se muestra también como una estrategia funcional ante una situación que puede comportar tanta afectividad negativa como es un resultado en contra. Sin embargo, hay estudios que contradicen estos resultados, indicando que ante situaciones de ira y enojo la distracción no se asocia a una regulación satisfactoria de estas emociones (Rivers, Brackett, Katulak y Salovey, 2007).

Observamos que a nivel grupal, el contagio emocional es percibido de forma muy positiva por los jugadores y en cambio, la individualidad ante los resultados adversos tiene una valoración muy negativa. Los jugadores perciben la individualidad como una forma de no hacer nada ante el resultado adverso y por lo tanto consideran que es perjudicial para la reacción del equipo.

De acuerdo con todo lo comentado hasta el momento, entendemos que la regulación emocional tiene un importante componente individual y colectivo. Pero además, la figura del entrenador resulta también imprescindible en la regulación emocional de los jugadores y en el rendimiento del equipo y el éxito de sus jugadores (Ramírez, 2002).

Los resultados revelan que su capacidad para cambiar la situación desfavorable durante el partido, es percibida por los jugadores como funcional ante resultados adversos, al igual que sucede con la inducción del contagio emocional positivo hacia los jugadores. Por el contrario, la no regulación muestra diferencias significativas respecto a estas dos estrategias. Ya se ha comentado en estas mismas conclusiones la importancia que la relación interpersonal tiene en la regulación de los estados emocionales. No es de extrańar que los estudios sobre los entrenadores con una sólida reputación, los definan como personas cálidas que disfrutan del contacto humano con una importante madurez emocional y que ejercen un estilo cooperativo (Ramírez, 2002). Cada vez se observa más literatura referente al impacto de los programas de coaching educativo para entrenadores (p. ej. Jones, Armour y Potrac, 2004; Jones y Turner, 2006). De acuerdo con dichos estudios han profundizado respecto al hecho de dar sentido a las realidades cotidianas de la práctica y entender mejor las relaciones que se establecen entre jugadores y entrenadores y entre los mismos jugadores (Potrac y Jones, 2010). Todos estos estudios han enfatizado la conexión existente entre las experiencias emocionales de los entrenadores y el tipo de rol que estos asumen, así como el tipo de entrenamiento que realizan y su relación con los jugadores (Jones y Turner, 2006; Potrac y Jones, 2010).

La sintonía emocional que el entrenador establezca con los jugadores resultara clave para fomentar un clima emocional 
positivo en el equipo. Como ya se ha comentado, el clima emocional dentro de un equipo es básico, ya que las emociones sentidas por ser miembro de un determinado equipo afectaran a las relaciones interpersonales que se establezcan en el mismo (Mackie y Smith, 2002). En este sentido, el entrenador tiene la responsabilidad de cohesionar al grupo, favoreciendo la experimentación de emociones positivas. Cuando las personas se sienten alegres las opiniones adoptan también un sentido más positivo, hecho que favorece un optimismo hacia la consecución de las metas (Jiménez y Domínguez, 2009).

\section{Conclusiones}

Cada vez son más los expertos que insisten en la importancia de que el deporte se estudie desde una perspectiva compleja (Seirul.lo, 2003) enfatizando la incorporación de diferentes componentes en la practica deportiva, destacando los físicos, técnicos, tácticos y psicológicos.

La importante conexión que se produce entre las emociones, la cognición y la fisiología (Damasio, 2010) y como ello influye en el rendimiento (Hanin, 2000a) nos lleva a considerar la importancia de incorporar el componente emocional a los entrenamientos. La necesidad de ayudar a los deportistas a ser más conscientes de sus estados emocionales y como afec- tan la intensidad de las emociones a nivel fisiológico deben facilitar la consecución de su rendimiento óptimo (Hanin, 2000a). Para ello, los entrenadores también requieren de una formación específica en este sentido ya que el tipo de relación que establecen con sus jugadores y el tipo de entrenamientos depende en gran medida de sus propias experiencia emocional (Potrac y Jones, 2010). En este sentido, contemplar la función que la figura del entrenador tiene en la evaluación de las situaciones que puedan generar emociones negativas, en el clima emocional del equipo y en la regulación emocional de sus jugadores resulta imprescindible para la mejora del rendimiento.

Entrenar la modulación de los estados emocionales debe favorecer la experimentación de estados de "Flow" durante el partido, entendiendo estos estados como los de máximo rendimiento de un jugador (López-Torres, Torregrosa y Roca, 2007). Alcanzar el nivel de intensidad emocional justo permitirá un nivel óptimo de rendimiento; para ello y especialmente en los deportes colectivos, debemos tener en cuenta la regulación emocional desde las perspectivas intra e interpersonales. La reevaluación cognitiva y el contagio emocional positivo grupal se muestran como las estrategias percibidas por los jugadores como más funcionales para poder afrontar resultados adversos.

\section{Referencias}

1. Arnold, M.B. (1960). Emotion and Personality: Psychological Aspects (Vol. 1). New York: Columbia University Press.

2. Balluerka, N. y Vergara, A.I. (2002). Diseños de investigación experimental en psicología. Madrid: Prentice-Hall.

3. Beck, A.T. (1976). Cognitive therapy and the emotional disorders. New York: Internacional University Press.

4. Bonanno, G.A. (2001). Emotion self-regulation. En T.J. Mayne y G.A. Bonanno (Eds.), Emotions: Current issues and future directions (pp. 251285). New York: Guilford Press.

5. Cabello González, R.; Fernández-Berrocal, P.; Ruiz-Aranda, D. y Extremera, N. (2006). Una aproximación a la integración de diferentes medidas de regulación emocional. Ansiedad y Estrés, 12, 155-166.

6. Coan, J.A. (2008). Toward a neuroscience of attachment. En J. Cassidy y P. R. Shaver (Eds.), Handbook of attachment: Theory, research and clinical applications (2nd ed., pp. 241-265). New York: Guilford Press.

7. Damasio, A. (2010). Y el cerebro creó al hombre: ¿Cómo pudo el cerebro generar emociones, sentimientos, ideas y el yo?. Barcelona: Destino.

8. Eisenberg, N. y Spinrad, T.L. (2004). Emotion-related regulation: Sharpening the definition. Child Development, 75 , 334-339.

9. Emmons, R.A. y Shelton, C.M. (2002). Gratitude and the science of positive psychology. En C. R. Synder y S. J. Lopez (Eds.), Handbook of positive psychology (pp. 459-471). New York, NY: Oxford University Press.

10. Estrada, O. y Pérez, E. (2008). Palabras e imágenes positivas en la respuesta de ansiedad en deportistas de competición. Cuadernos de Psicologia del Deporte, 8, (1) 31-45.

11. Feltz, D.; Lirgg, C. y Albrecht, R. (1992). Psychological implications of competitive running in elite young distance runners: A longitudinal analysis. The Sport Psychologist, 6, 128-138.

12. Folkman, S. y Lazarus, R.S. (1985). If it changes it must be a process:
Study of emotion and coping during three stages of a college examination. Journal of Personality and Social Psychology, 48, 150-170.

13. Forgas, J.P. y Ciarocchi, J. (2002). On managing moods: Evidence for the role of homeostatic cognitive strategies in affect regulation. Personality and Social Psychology Bulletin, 28, 336-345.

14. Fredrickson, B. L. y Joiner, T. (2002). Positive emotions trigger upward spirals toward emotional well-being. Psychological Science, 13, 172-175.

15. Frijda, N.H. (1986). The emotions. Cambridge: Cambridge University Press.

16. Frijda, N.H.; Markam, S.; Sato, K. y Wiers, R. (1995). Emotions and emotion words. En J. A. Russell, J. M. Ferdandez-Dols, A. S. R. Manstead y J. C. Wellenkamp (Eds.), Everyday conceptions of emotion (pp. 121-143). Dordrecht, the Netherlands: Kluwer Academic Publishers.

17. Goldsmith, H.H. y Davidson, R.J. (2004). Disambiguating the components of emotion regulation. Child Development, 75, 361-365.

18. Gould, D. y Weinberg, R. (1985). Sources of worry in successful and less successful intercollegiate wrestlers. Journal of Sport Behavior, 8, $115-127$.

19. Gross, J.J. (1998). The Emerging Field of Emotion Regulation: An integrative Review. Review of General Psychology, 2, 271-299.

20. Gross, J.J. y John, O.P. (2003). Individual differences in two emotion regulation processes: Implications for affect, relationships, and wellbeing. Journal of Personality and Social Psychology, 85, 348-362.

21. Gross, J.J. y Thompson, R. (2007). Emotion regulation: Conceptual foundations. In J.J. Gross (Ed.), Handbook of Emotion Regulation (pp. 3-24). New York: Guilford Press.

22. Hanin, Y.L. (1980). A study of anxiety in sports. In W. F. Straub (Ed.), Sport psychology: An analysis of athlete behavior (pp. 236-249). Ithaca, NY: Mouvement. 
23. Hanin, Y.L. (1986). State-trait anxiety research on sports in the USSR. En C. D. Spielberger y R. Diaz-Guerrero (Eds.), Cross cultural anxiety, Vol. 3 (pp. 45-64). Washington: Hemisphere.

24. Hanin, Y.L. (1997). Emotions and athletic performance: Individual Zones of Optimal Functioning model. European Yearbook of Sport Psychology, 1, 29-72.

25. Hanin, Y.L. (2000a). IZOF-based emotions-profiling: Step-wise procedures and forms. En Y.L. Hanin (Ed.), Emotions in sport (pp. 303-313). Champaign, IL: Human Kinetics.

26. Hanin, Y.L. (2000b). Individual zones of optimal functioning (IZOF) model: Emotion-performance relationships in sport. En Y.L. Hanin (Ed.), Emotions in sport (pp. 65-89). Champaign, IL: Human Kinetics.

27. Hanin, Y.L. (2000c). Successful and poor performance and emotions. In Y.L. Hanin (Ed.), Emotions in sport (pp.157-187). Champaign, IL: Human Kinetics.

28. Hardy, L., Mullen, R. y Jones, G. (1996). Knowledge and conscious control of motor actions under stress. British Journal of Psychology, 87, 621-636.

29. Isen, A. M. y Means, B. (1983). The influence of positive affect on decision-making strategy. Social Cognition, 2, 18-31

30. Isen, A. M., Rosenzweig A.S. y Young M.J. (1991). The influence of positive affect on clinical problem solving. Medical Decision Making. 11, 221-7.

31. Jiménez Sánchez, M. y Domínguez Sánchez, F. (2009). Cerebro y humor. En Fernández Abascal, E. comp. (2009). Emociones Positivas. Madrid: Ediciones Piràmide

32. Jones, G. y Hanton, S. (2001). Pre-competitive feeling states and directional anxiety interpretations. Journal of Sports Sciences, 19, 385-395.

33. Jones, G. y Hardy, L. (1990). Stress in sport: Experiences of some elite performers. In G. Jones \& L. Hardy (Eds.), Stress and performance in sport (pp. 247-277). Chichester: John Wiley.

34. Jones, R. L.; Armour, K. M. y Potrac, P. (2004) Sports coaching cultures: from practice to theory London: Routledge.

35. Jones, R. L. y Turner, P. (2006). Teaching coaches to coach holistically: the case for a problem based learning (PBL) approach. Physical Education and Sport Pedagogy, 11(2), 181-202.

36. Kroll, W. (1980). The stress of high performance athletics. En P. Klavora y V. Daniel (Eds.), Coach, athlete, and the sport psychologist (2nd ed.). Champaign: Human Kinetics.

37. Langston, C.A. (1994). Capitalizing on and coping with daily-life events: expressive responses to positive events. J. Personal. Soc. Psychol., 67, 1112-1125.

38. Larsen, R. y Prizmic, Z. (2008). The regulation of emotional wellbeing: Overcoming the hedonic treadmill. En M. Eid y R. Larsen (Eds.), The science of subjective well-being (pp. 258-289). Nueva York: Guilford Press.

39. Lazarus, R.S. (1968). Psychological Stress and the Coping Processes. New York: McGraw Hill

40. Lazarus, R.S. (1991). Emotion and adaptation. New York: Oxford University Press.

41. Lazarus, R. S. (1999). Stress and emotion: A new synthesis. London: Free Association Books.

42. Lazarus, R. (1996). The role of coping in the emotions and how coping changes over the life course. En C. Magai y SH McFadden (Eds), Handbook of emotion, adult development and aging (pp. 289-306). San Diego, CA: Academic Press.

43. Levenson, R. W. (1988). Emotion and the Autonomic Nervous System: A Prospectus for Research on Autonomic Specificity. In H. L. Wagner (Ed.) Social Psychophysiology and Emotion: Theory and Clinical Application, pp 17-42. Hoboken, NJ: John Wiley \& Sons.
44. López-Torres, M.; Torregrosa, M. y Roca, J. (2007). Características del flow, ansiedad y estado emocional, en relación con el rendimiento de deportistas de elite. Cuadernos de Psicología del Deporte, 7 (1), 25-44.

45. Mackie, D.M. y Smith, E.R. (2002). Intergroup emotions and the social self: Prejudice reconceptualized as diferentiated reactions to outgroups. En J. P. Forgas y K. D. Williams (Eds.), The social self: Cognitive, interpersonal, and intergroup perspectives (pp. 309-326). Philadelphia, PA: Psychology Press.

46. Márquez, S. (2004). Ansiedad, estrés y deporte. Madrid: EOS.

47. Mesquita, B. y Ellsworth, P.C. (2001). The role of culture in appraisal. En K.R. Scherer, A. Schorr y T. Johnstone (Eds.), Appraisal Processes in Emotion (pp. 233-248). New York: Oxford University Press.

48. Palmero, F.; Díez, J.L. y Breva, A. (2001). Type a Behavior Pattern Today: Relevance of the Jas-S Factor to Predict Heart Rate Reactivity. Behavioral Medicine, 27, 28-36

49. Pargman, D. (1986). Stress and motor performance: Understanding and coping. Tallahassee, FL:

50. Potrac, P. y Jones, R. (2010). Power in coaching. En R. Jones, P. Potrac, C. Cushion, y L.T Ronglan (Eds), The sociology of sports coaching. London: Routledge.

51. Richards, J. y Gross, J. (2000). Emotion Regulation and Memory: the cognitive costs of keeping one's cool. Journal of Personality and Social Psychology, 79 (3) , pp410-424.

52. Ramírez, M.C. (2002). Caracterización del entrenador de alto rendimiento deportivo. Cuadernos de psicología del deporte, 2, 15-37.

53. Rimé, B. (2009). Emotion elicits the social sharing of emotion: Theory and empirical review. Emotion Review, 1, 60-85.

54. Rimé, B. (2012). El compartimiento social de las emociones. Bilbao: Desclée de Brouwer.

55. Rivers, S., Brackett, M. A., Katulak, N. y Salovey, P. (2007). Regulating anger and sadness: An exploration of discrete emotions in emotion regulation. Journal of Happiness Studies, 8, 393-427.

56. Roseman, I.J. (1984). Cognitive determinants of emotion: A structural theory. En P. Shaver (Ed.), Review of personality and social psychology: Vol. 5 Emtions, relationships and health (pp. 11-36). Beverly Hills, CA: Sage.

57. Scherer, K.R. (1997). Profiles of emotion-antecedent appraisal: Testing theoretical predictions across cultures. Cognition and Emotion, 11, 113150.

58. Seirul.lo, F. (2003). Sistemas Dinámicos y Rendimiento en Deportes de Equipo. 1st Meeting of Complex Systems and Sport. INEFC-Barcelona.

59. Siegman, A.W. y Smith, T.W. (1994). Anger, hostility and health. Hillsdale, N.J.: Lawrence Erlbaum Associates.

60. Siemer, M.; Mauss, I. y Gross, J.J. (2007). Same situation--different emotions: How appraisals shape our emotions. Emotion, 7(3), 592-600.

61. Smith, C.A. y Ellsworth, P.C. (1985). Patterns of cognitive appraisal in emotions. Journal of Personality and Social Psychology, 48, 813-838.

62. Weiner, B. (1982). The emotional consequences of causal attributions. En M.S. Clark y S.T. Fiske (Eds.), Affect and cognition (pp. 185-209). The 7th Annual Carnegie Symposium on Cognition. Hillsdale, NJ: Lawrence Erlbaum Associates Inc.

63. Weiner, B. (1986). An attributional theory of motivation and emotion. New York: Springer.

64. Zautra, A.J. (2003). Emotions, Stress, and Health. New York: Oxford University Press.

65. Zautra, A.J.; Affleck, G.G.; Tennen, H.; Reich J.W. y Davis M.C. (2005) Dynamic Approaches to Emotions and Stress in Everyday Life: Bolger and Zuckerman Reloaded With Positive as Well as Negative Affects. Journal of Personality. 73(6), 1511-1538. 